\title{
oints
} $\sqrt{2}$

\section{The role of arthroscopic capsulo-labral repair in unidirectional post-traumatic shoulder instability in adolescent athletes participating in overhead or contact sports}

\author{
GIACOMO DELLE ROSE, MARIO BORRONI, ALESSANDRO CASTAGNA
}

Shoulder Department, IRCCS Humanitas Institute, Rozzano, Milan, Italy

\begin{abstract}
Purpose: this study was performed to identify the role of arthroscopic capsulo-labral repair (ACR) in unidirectional post-traumatic shoulder instability in adolescent athletes participating in overhead or contact sports.

Methods: sixty-five adolescent patients (aged 13 to 18 years) with post-traumatic shoulder instability submitted to arthroscopic surgery were selected from our database. The mean follow-up duration was 63 months. Shoulder range of motion and functional outcomes were evaluated preoperatively and postoperatively using the Single Assessment Numeric Evaluation (SANE), Rowe, and American Shoulder and Elbow Surgeons (ASES) scores. In addition, details in the database on the type of sport practiced, time until surgery, and number of dislocations were analyzed to look for possible correlations with the recurrence rate.

Results: at the final follow-up, the mean SANE score was $87.23 \%$ (range: $30 \%$ to $100 \%$ ) (preoperative mean score: $46.15 \%$ [range, $20 \%$ to $50 \%$ ]); the mean Rowe score was 85 (range: 30 to 100) (preoperative mean score: 35.9 [range: 30 to 50]); and the mean ASES score was 84.12 (range: 30 to 100 ) (preoperative mean score: 36.92 [range: 30 to 48]). Mean forward flexion and external rotation with the arm at $90^{\circ}$ abduction did not show changes compared with preoperative values; $81.5 \%$ of the patients returned to their
\end{abstract}

\footnotetext{
Corresponding Author:

Giacomo Delle Rose, MD

Shoulder Department, IRCCS Humanitas Institute,

Rozzano, Milan, Italy

E-mail: drjames@alice.it
}

pre-injury level of sports activities, and the failure rate was $21.5 \%$. The recurrence rate was not related to the postoperative scores $(\mathrm{p}=0.556$ for SANE, $\mathrm{p}=0.753$ for Rowe, and $p=0.478$ for ASES), number of preoperative episodes of instability $(\mathrm{p}=0.59)$, or time that elapsed between the first instability episode and the surgery $(p=0.43)$. A statistically significant association $(\mathrm{p}=0.0021)$ was found between recurrence and the type of sport practiced.

Conclusions: ACR is a reasonable surgical option in an adolescent population participating in sports. It has a role in restoring shoulder stability with very low morbidity; however, the failure rate is higher than in the adult population and both the young patients and their relatives must be properly informed about the expected outcome of the procedure.

Level of Evidence: level IV, therapeutic case series.

Key Words: adolescents, athletes, arthroscopy, instability, shoulder.

\section{Introduction}

Traumatic anterior glenohumeral $(\mathrm{GH})$ dislocations are commonly seen in children and adolescents participating in contact or overhead sports (1). In particular, reported rates of recurrence after the initial injury may reach $90-95 \%$ in patients younger than 25 years of age (2). One study reported a failure rate of $21.4 \%$ in a skeletally immature population (aged from 10 to 13 years) (3).

Arthroscopic capsulo-labral repair (ACR) of acute GH dislocations in young athletes has been shown to decrease the incidence of recurrent shoulder instability compared with non-operative treatment (4). We have 
found only one article in the literature reporting the results of ACR in adolescents participating in overhead or contact sports; moreover, these subjects' level of sporting activity was not clearly defined (5).

The aim of this study was to determine the efficacy and role of ACR in unidirectional post-traumatic shoulder instability in adolescent athletes participating in overhead or contact sports. Our hypothesis is that it is an effective procedure.

\section{Methods}

\section{Patient selection}

This is a retrospective cohort study. Patients were identified from a database of 650 consecutive ACRs for recurrent anterior shoulder instability, performed between September 2002 and December 2005. One hundred and twenty-six adolescents aged between 13 and 18 years were identified according to the age limits defined by the American Academy of Pediatrics. Patients with significant glenoid bone loss (25\%), as evaluated arthroscopically according to Burkhart et al. (6), and patients with connective tissue pathology, such as those with Marfan syndrome, Ehlers-Danlos syndrome, or Down syndrome, or a family history of connective tissue pathology (a total of 12 patients) were excluded from the study. From the remaining 114 adolescent patients, only those who practiced overhead or contact sports at a competitive level were selected. Competitive level was defined as regular participation, individually or as part of a team, in regional or national level matches or tournaments, and affiliation to national sports federations. In total, 67 cases met the inclusion criteria. Two patients were dropped from the cohort because they could not be traced. The remaining 65 patients were contacted and invited to participate in a study-specific outcome evaluation. The sports practiced by these patients were tennis, basketball, volleyball, water polo, swimming, rugby, and soccer (goalkeeper).

\section{Preoperative evaluation}

The patients' charts were reviewed in our office to establish details of their personal and medical history, including age, sex, sport practiced, date of first dislocation or subluxation, and total number of disloca- tions or subluxations prior to the surgery. The sample comprised 44 males and 21 females with a mean age of 16 years (range: 13-18 years) at the time of surgery. In all cases, a traumatic injury, sustained during sports activities, was indicated as responsible for the first episode of shoulder instability. Fifty-three patients had a history of shoulder dislocation, whereas the other 12 described a history consistent with shoulder subluxation. Dislocation was defined as GH instability that required manual reduction, while subluxation was defined as an episode in which the humerus translated anteriorly, causing a brief period of pain that soon resolved and did not require a formal medical intervention. The mean time between the injury and the surgery was 17.6 months (range: 9 to 28 months). Preoperatively, a single physician evaluated all patients, measuring their shoulder range of motion with a goniometer and administering an outcomes questionnaire, which included a number of validated scores for the measurement of shoulder status. In particular, the Rowe score, American Shoulder and Elbow Surgeons (ASES) score, and Single Assessment Numeric Evaluation (SANE) score (7) were determined.

Although these scores provide similar information, the Rowe and ASES scores were included because the relevant data are systematically recorded in our shoulder department's data files.

\section{Surgical procedures}

All the procedures were performed with the patient in the lateral decubitus position under regional anesthesia. At the beginning of each procedure, glenoid bone loss was measured in accordance with Burkhart et al. (6) and recorded in the database.

The Spectrum hook system (ConMed Linvatec, Largo, FL) was used to perform the capsule-labral repair, which was determined by the type of injury found at the time of arthroscopy.

In five cases the labrum was found to be attached to the glenoid rim, and just a re-tensioning of the capsulo-labral complex was performed. After the capsule had been abraded, capsular plication with absorbable No. 1 polydioxanone suture (PDS; Ethicon Somerville, $\mathrm{NJ}$ ) was performed to achieve the desired tension of the anterior band of the inferior GH ligament and middle GH ligament and one or two posterior stitches 
were applied to tension the posterior band of the inferior GH ligament.

An anterior labral periosteal sleeve avulsion lesion was found in 19 cases and an anterior Bankart lesion in 41 cases. These lesions were repaired with a suture anchor technique as described by Snyder et al. (8). In all these cases we used double-loaded absorbable suture anchors, and in each procedure at least four sutures were placed anteriorly.

\section{Postoperative care}

After surgery, patients were immobilized for four weeks by means of a shoulder sling with the arm in $15^{\circ}$ of abduction and $15^{\circ}$ of external rotation.

Starting from the second postoperative week, free active ipsilateral elbow and wrist movements were permitted with the arm at the side of the body. Active-assisted and passive shoulder abduction was not allowed for four weeks. External rotation of the arm to neutral with the elbow flexed to $90^{\circ}$ was encouraged for activities of daily living such as writing or typing. Use of the shoulder immobilizer was discontinued four weeks after the operation, and a home-based program of active and active-assisted range-of-motion exercises focusing on forward flexion, abduction, and external rotation was initiated. At eight weeks postoperatively, formal strengthening exercises for the rotator cuff, deltoid and periscapular muscles were started. Patients were allowed to resume unrestricted sports activities five months postoperatively if range of motion and shoulder muscle strength and endurance had returned to normal.

\section{Postoperative assessment}

Patients were clinically reviewed at a mean of 63 months (range: 51 to 92 months) after surgery. All 65 patients returned to our office for a comprehensive evaluation including a physical examination, measurement of range of motion, and completion of the outcome assessment with recording of shoulder scores.

The same physician who had examined patients preoperatively performed all the follow-up evaluations, focusing specifically on the presence of complications, return to the pre-injury level of sporting activity, and recurrence (dislocation or subluxation).

\section{Data analysis}

Statistical analysis with the Mann-Whitney U-test was performed to investigate the relationship between recurrence and preoperative scores (ASES, SANE, Rowe), type of sport practiced, number of preoperative dislocations, and time from the first episode of instability. The difference was significant when $p$ $<0.05$.

\section{Results}

No intraoperative or postoperative surgical complications were reported in this group of patients. In all 65 patients, at follow-up, the mean SANE score was $87.2 \%$ (range: $30 \%$ to $100 \%$ ) (preoperative mean, $46.1 \%$ [range: $20 \%$ to $50 \%$ ]), the mean Rowe score was 85 (range, 30 to 100) (preoperative mean, 35.9 [range, 30 to 50]), and the mean ASES score was 84.1 (range, 30 to 100) (preoperative mean, 36.9 [range, 30 to 48]).

Mean postoperative forward flexion and external rotation with the arm at $90^{\circ}$ of abduction did not change from the preoperative mean values of $180^{\circ}$ and $86^{\circ}$, respectively.

Of the 65 patients, $53(81.5 \%)$ returned to their preinjury level of sporting activity. The other 12 resumed their sports at a lower level because they lacked confidence in the repaired shoulder.

Fourteen $(21.5 \%)$ of the 65 patients had recurrence (including dislocations and subluxations) of GH instability. This was due to significant trauma in seven patients, whereas in the other seven the recurrence occurred without a specific traumatic event.

There was no evidence of a difference in clinical scores between the patients who had recurrence of GH dislocation and those who did not $(\mathrm{p}=0.556 ; \mathrm{p}=0.753$; and $\mathrm{p}=0.478$ for the SANE, Rowe, and ASES scores, respectively).

With regard to postoperative recurrence and sports practiced, the recurrence rate was $40 \%$ in the water polo players, $33.3 \%$ in the rugby players, $25 \%$ in the basketball players, $20 \%$ in the soccer players, $16.6 \%$ in the tennis players, $15.3 \%$ in the volleyball players, and $12.5 \%$ in the swimmers, and these data were statistically significant $(\mathrm{p}=0.0021)$. 
The mean number of instability episodes prior to surgery was 3.3 (116 dislocations and 59 subluxations) in the non-recurrence group and 1.3 (13 dislocations and five subluxations) in the recurrence group $(\mathrm{p}=0.59)$. The mean time from the first episode until the surgical procedure was 17.7 months in the non-recurrence group and 18.7 months in the recurrence group $(\mathrm{p}=$ $0.43)$.

\section{Discussion}

The literature contains numerous articles reporting the efficacy of ACR in adults participating in overhead or contact sports $(9,10)$. Few articles have reported the results of ACR in adolescent populations $(2,11)$. Jones et al. (5) reported the results of ACR in adolescents participating in overhead or contact sports but the level of their involvement in these sports was not clearly defined (5). The authors reported a $18.7 \%$ recurrence rate after ACR in adolescents; $84 \%$ of athletes who underwent ACR (11 out of 13) resumed their sports activities at the pre-injury level. These results were slightly better than those reported in the present study in terms of recurrence rate $(18.7 \%$ vs $21.5 \%)$ and percentage of athletes that resumed their sporting activities at their pre-injury level $(81.5 \%$ vs $84 \%)$. This could be due to our group of patients practicing sports at a higher level.

We analyzed different covariates in order to find an explanation for the high failure rate: baseline scores, type of sport practiced, number of dislocations, and time after the first episode of GH dislocation. Only the type of sport was statistically correlated with recurrence. In particular, our data seem to report a higher recurrence rate in very high-energy contact sports (rugby) and high-energy contact sports associated with overhead position of the arm (water polo).

This study has several limitations. Even though the follow-up time was long (63 months), it is a retrospective cohort study and the sports practiced were not equally distributed in the population.

However, it is important to underline that this is the first study of a true population of adolescent athletes. Furthermore, all the subjects were treated with the same surgical procedure, performed by the same sur- geon, and followed the same postoperative rehabilitation program, supervised by the same physician. Finally, despite the homogeneity of the sample (all the patients practiced sports, and selection was performed according to the definition of the American Academy of Pediatrics), its size was not large enough to attempt a prognostic analysis.

In conclusion, ACR is a reasonable surgical option in an adolescent population participating in different sports. It has a role in restoring shoulder stability with very low morbidity; however, the failure rate is higher than in the adult population and both the young patients and their relatives must be properly informed about the expected outcome of the procedure.

\section{References}

1. Good CR, MacGillivray JD. Traumatic shoulder dislocation in the adolescent athlete: advances in surgical treatment. Curr Opin Pediatr 2005;17:25-29.

2. Deitch J, Mehlman CT, Foad SL, Obbehat A, Mallory M. Traumatic anterior shoulder dislocation in adolescents. Am J Sports Med 2003;31:758-763.

3. Cordischi K, Li X, Busconi B. Intermediate outcomes after primary traumatic anterior shoulder dislocation in skeletally immature patients aged 10 to 13 years. Orthopedics 2009;32: 728-734.

4. Bottoni CR, Wilckens JH, DeBerardino TM, et al. A prospective, randomized evaluation of arthroscopic stabilization versus nonoperative treatment in patients with acute, traumatic, first-time shoulder dislocations. Am J Sports Med 2002; 30: 576-580.

5. Jones KJ, Wiesel B, Ganley TJ, Wells L. Functional outcomes of early arthroscopic Bankart repair in adolescents aged 11 to 18 years. J Pediatr Orthop 2007;27:209-213.

6. Burkhart SS, DeBeer JF, Tehrany AM, Parten PM. Quantifying glenoid bone loss arthroscopically in shoulder instability. Arthroscopy 2002;18:488-491.

7. Williams GN, Gangel TJ, Arciero RA, Uhorchak JM, Taylor DC. Comparison of the Single Assessment Numeric Evaluation method and two shoulder rating scales. Outcomes measures after shoulder surgery. Am J Sports Med 1999; 27:214-221.

8. Snyder SJ, Banas MP, Belzer JP. Arthroscopic treatment of anterior shoulder instability using threaded suture anchors and nonabsorbable suture. Instr Course Lect 1996;45:71-81.

9. Ide J, Maeda S, Takagi K. Arthroscopic Bankart repair using suture anchors in athletes: Patient selection and postoperative sports activity. Am J Sports Med 2004;32:1899-1905.

10. Mazzocca AD, Brown FM Jr, Carreira DS, Hayden J, Romeo AA. Arthroscopic anterior shoulder stabilization of collision and contact athletes. Am J Sports Med 2005;33:52-60.

11. Lawton RL, Choudhury S, Mansat P, Cofield RH, Stans AA. Pediatric shoulder instability: Presentation, findings, treatment, and outcomes. J Pediatr Orthop 2002;22:52-61. 